\title{
On the Multiplicity of Solutions for the Discrete Boundary Problem Involving the Singular $\phi$-Laplacian
}

\begin{abstract}
Zihua Qiu
School of Mathematics and Information Science, Guangzhou University, Guangdong Guangzhou 510006, China

Correspondence should be addressed to Zihua Qiu; zihuaqiu@gzhu.edu.cn

Received 19 August 2021; Revised 24 September 2021; Accepted 29 September 2021; Published 13 October 2021

Academic Editor: Fanglei Wang

Copyright ( 2021 Zihua Qiu. This is an open access article distributed under the Creative Commons Attribution License, which permits unrestricted use, distribution, and reproduction in any medium, provided the original work is properly cited.

In this paper, we consider the multiplicity of solutions for a discrete boundary value problem involving the singular $\phi$-Laplacian. In order to apply the critical point theory, we extend the domain of the singular operator to the whole real numbers. Instead, we consider an auxiliary problem associated with the original one. We show that, if the nonlinear term oscillates suitably at the origin, there exists a sequence of pairwise distinct nontrivial solutions with the norms tend to zero. By our strong maximum principle, we show that all these solutions are positive under some assumptions. Moreover, the solutions of the auxiliary problem are solutions of the original one if the solutions are appropriately small. Lastly, we give an example to illustrate our main results.
\end{abstract}

\section{Introduction}

Let $Z$ and $R$ denote the sets of integers and real numbers, respectively. For $a, b \in Z$, define $Z(a)=\{a, a+1, \cdots\}$ and $Z($ $a, b)=\{a, a+1, \cdots, b\}$ when $a \leq b$.

In this paper, we consider the following boundary value problem of prescribed mean curvature equations in Minkowski spaces:

$$
\left\{\begin{array}{l}
\nabla\left(\frac{\Delta u_{k}}{\sqrt{1-\left(\Delta u_{k}\right)^{2}}}\right)+\lambda f\left(k, u_{k}\right)=0, \quad k \in Z(1, T), \\
u_{0}=\alpha u_{1}, \quad u_{T+1}=0,
\end{array}\right.
$$

where $T$ is a given positive integer, $\alpha$ is a constant in $[0,1], \nabla$ is the backward difference operator defined by $\nabla u_{k}=u_{k}$ $u_{k-1}, \Delta$ is the forward difference operator defined by $\Delta u_{k}=$ $u_{k+1}-u_{k}$, and $f(k, \cdot) \in C(R, R)$ for each $k \in Z(1, T)$.
In 2019, Chen et al. in [1] considered problem (1) in the case where $f(k, x)=\mu_{k} x^{q}$ and $\alpha=1$, that is,

$$
\left\{\begin{array}{l}
\nabla\left(\frac{\Delta u_{k}}{\sqrt{1-\left(\Delta u_{k}\right)^{2}}}\right)+\lambda \mu_{k}\left(u_{k}\right)^{q}=0, \quad k \in Z(1, T), \\
\Delta u_{0}=0=u_{T+1} .
\end{array}\right.
$$

By using upper and lower solutions, the Brouwer degree theory, and Szulkin's critical point theory for convex, lower semicontinuous perturbations of $C^{1}$-functions, the authors obtained the intervals of the parameter $\lambda$ such that problem (2) has zero, one, or two positive solutions. Earlier in 2008, Bereanu and Mawhin in [2] obtained the existence of at least one or two solutions for the boundary value problems of second-order nonlinear differences with singular $\phi$-Laplacian by using the Brouwer degree together with fixed point reformulations. For the existence and multiplicity of positive solutions of the associated differential problems to (1), we 
refer to $[3,4]$. And for the boundary value problems of nonsingular differential equations, we refer to [5-9].

Difference equations arise in various research fields. For the existence and multiplicity of solutions of boundary value problems of difference equations, the classical methods are fixed point theory, the method of upper and lower solution techniques, Rabinowitz's global bifurcation theorem, etc. (see $[2,10,11])$. Since 2003, variational methods have been employed to study difference equations [12], by which various results are obtained. See, for example, periodic solutions and subharmonic solutions [13, 14], homoclinic solutions [15-22], heteroclinic solutions [23], and boundary value problems [24-27]. In recent years, boundary value problems of difference equations involving $\phi$-Laplacian have aroused extensive attention from scholars; for example, in 2019, Zhou and Ling in [28] considered the following Dirichlet problem of the second-order nonlinear difference equation:

$$
\left\{\begin{array}{l}
-\Delta\left(\phi_{c}\left(\Delta u_{k-1}\right)\right)=\lambda f\left(k, u_{k}\right), \quad k \in Z(1, T), \\
u_{0}=u_{T+1}=0
\end{array}\right.
$$

where $\phi_{c}$ is the mean curvature operator defined by $\phi_{c}(s)$ $=s / \sqrt{1+s^{2}}$. The authors obtained the existence of infinitely many positive solutions for problem (3). The authors in [29] extended the results of [28] to the following Dirichlet problem:

$$
\left\{\begin{array}{l}
-\Delta\left(\phi_{c}\left(\Delta u_{k-1}\right)\right)+q_{k} \phi_{c}\left(u_{k}\right)=\lambda f\left(k, u_{k}\right), \quad k \in Z(1, T), \\
u_{0}=u_{T+1}=0 .
\end{array}\right.
$$

For the Robin problem of the second-order nonlinear difference equation,

$$
\left\{\begin{array}{l}
-\Delta\left(\varphi_{p}\left(\Delta u_{k-1}\right)\right)+q_{k} \varphi_{p}\left(u_{k}\right)=\lambda f\left(k, u_{k}\right), \quad k \in Z(1, T), \\
\Delta u_{0}=u_{T+1}=0
\end{array}\right.
$$

where $\varphi_{p}$ is a special $\phi$-Laplacian operator [13] defined by $\varphi_{p}(s)=p|s|^{p-2} s / 2 \sqrt{1+|s|^{p}}$ with $p \geq 2$; we refer to [30].

Up to now, there is less work on the boundary value problems of difference equations involving the singular $\phi$ Laplcian; the known results are the existence of at least one or two solutions. The aim of this paper is to obtain the existence of infinitely many solutions for problem (1), and problem (1) contains the Dirichlet problem (when $\alpha=0$ ) and Robin problem (when $\alpha=1$ ) as special cases. The difficulty lies that the domain of the singular $\phi$-Laplcian is a finite open interval $(-a, a)$, not $(-\infty,+\infty)$. The tool is a critical point result in [31]. However, we can not apply the critical point theory to the problem (1) directly, since the domain of the singular $\phi$-Laplcian in $(1)$ is $(-1,1)$; we need to extend the domain of the singular $\phi$-Laplcian to $(-\infty,+\infty)$. Instead, we consider the auxiliary problem (20) associated with problem (1) in Section 2. We will show that solutions of problem (20) are solutions of problem (1) if the solutions are appropriately small. For general background on difference equations, we refer the reader to monographs [32, 33].

This paper is organized as follows. In Section 2, an auxiliary problem associated with problem (1) is established, the variational framework associated with this auxiliary problem is established, and the abstract critical point theorem is recalled. In Section 3, our main results are presented. And we also establish a strong maximum principle and obtain the existence of infinitely many positive solutions for (1) according to the oscillating behavior of $f$ at the origin. Finally, in Section 4, an example is given to illustrate our main results.

\section{Preliminaries}

In this section, we will first introduce a lemma (Theorem 2.5 of [31]).

Let $X$ be a reflexive real Banach space, and let $I_{\lambda}: X$ $\longrightarrow R$ be a function satisfying the following structure hypothesis:

$(\Lambda) I_{\lambda}(u):=\Phi(u)-\lambda \Psi(u)$ for all $u \in X$, where $\Phi, \Psi: X$ $\longrightarrow R$ are two functions of class $C^{1}$ on $X$ with $\Phi$ coercive, i.e., $\lim _{\|u\| \longrightarrow+\infty} \Phi(u)=+\infty$, and $\lambda$ is a real positive parameter.

If $\inf _{X} \Phi<r$, let

$$
\begin{array}{r}
\mu(r):=\inf _{u \in \Phi^{-1}(-\infty, r)} \frac{\left(\sup _{v \in \Phi^{-1}(-\infty, r)} \Psi(v)\right)-\Psi(u)}{r-\Phi(u)}, \\
\delta:=\liminf _{r} \mu\left(\inf _{X} \Phi\right)^{+}
\end{array}
$$

Obviously, $\delta \geq 0$. When $\delta=0$, in the sequel, we agree to $\operatorname{read} 1 / \delta$ as $+\infty$.

Lemma 1. Assume that the condition $(\Lambda)$ holds, and $\delta<+\infty$; then, for each $\lambda \in(0,1 / \delta)$, the following alternative holds: either

$\left(a_{1}\right)$ there is a global minimum of $\Phi$ which is a local minimum of $I_{\lambda}$, or

$\left(a_{2}\right)$ there is a sequence $\left\{u_{n}\right\}$ of pairwise distinct critical points (local minima) of $I_{\lambda}$, with $\lim _{n \longrightarrow+\infty} \Phi\left(u_{n}\right)=\inf _{X} \Phi$, which weakly converges to the global minimum of $\Phi$.

We will use this lemma to investigate problem (1). Now, we establish the variational framework associated with problem (1). We consider the $T$-dimensional Banach space.

$$
S=\left\{u: Z(0, T+1) \longrightarrow R: u_{0}=\alpha u_{1}, u_{T+1}=0\right\}
$$

endowed with the norm

$$
\|u\|:=\left(\sum_{k=1}^{T}\left(\Delta u_{k}\right)^{2}\right)^{1 / 2}
$$


We consider another norm in $S$, that is,

$$
\|u\|_{\infty}:=\max \left\{\left|u_{k}\right|: k \in Z(1, T)\right\}
$$

For each $u \in S$, there exists a $\tau \in Z(1, T)$, such that

$$
\|u\|_{\infty}=\left|u_{\tau}\right|=\left|\sum_{k=\tau}^{T} \Delta u_{k}\right| \leq \sum_{k=1}^{T}\left|\Delta u_{k}\right| \leq T^{1 / 2}\|u\|
$$

thus,

$$
\|u\| \geq \frac{1}{T^{1 / 2}}\|u\|_{\infty}
$$

We mention that the equality in (11) holds if we let $u_{k}$ $=(T+1-k) c, k \in Z(1, T), u_{0}=\alpha T c$, where $c$ is a nonzero constant. In fact, in this case, $\|u\|=T^{1 / 2}|c|$ and $\|u\|_{\infty}=T|c|$.

We notice that the singular operator $\phi(s)=s / \sqrt{1-s^{2}}$ in problem (1) only defined for $s \in(-1,1)$. In order to use Lemma 1 , we need to extend the domain of the singular operator $\phi$ to $(-\infty, \infty)$. Take

$$
g(s)=\left\{\begin{array}{l}
\frac{s}{\sqrt{1-s^{2}}}, \quad|s| \leq \frac{\sqrt{3}}{2} \\
2 s, \quad|s|>\frac{\sqrt{3}}{2} .
\end{array}\right.
$$

Then, $g(s)$ is continuous in $(-\infty,+\infty)$, and the primary function of $g$ is given by

$$
G(s)=\left\{\begin{array}{l}
\frac{s}{1+\sqrt{1-s^{2}}}, \quad|s| \leq \frac{\sqrt{3}}{2} \\
s^{2}-\frac{1}{4}, \quad|s|>\frac{\sqrt{3}}{2}
\end{array}\right.
$$

We define

$$
\begin{aligned}
& \Phi(u)=\sum_{k=0}^{T} G\left(\Delta u_{k}\right)+\frac{\alpha G\left(\Delta u_{0}\right)}{1-\alpha} \\
& \Psi(u)=\sum_{k=1}^{T} F\left(k, u_{k}\right)
\end{aligned}
$$

for each $u \in S$, where $F(k, u)=\int_{0}^{u} f(k, \tau) d \tau$ for every $k \in Z($ $1, T)$. When $\alpha=1$, we read $\left(\alpha G\left(\Delta u_{0}\right)\right) /(1-\alpha)$ as 0 in (14), since

$$
\lim _{\alpha \longrightarrow 1} \frac{\alpha G\left(\Delta u_{0}\right)}{1-\alpha}=\lim _{\alpha \longrightarrow 1} \frac{\alpha(1-\alpha)^{2} u_{1}^{2}}{(1-\alpha)\left(1+\sqrt{ } 1-(1-\alpha)^{2} u_{1}^{2}\right.}=0 .
$$

Put

$$
I_{\lambda}(u)=\Phi(u)-\lambda \Psi(u)
$$

for $u \in S$. Then, $\Phi$ and $\Psi$ are two functionals of class $C^{1}(S, R)$ whose Gâteaux derivatives at the point $u \in S$ are given by

$$
\begin{aligned}
& \Phi^{\prime}(u)(v)=\sum_{k=0}^{T} g\left(\Delta u_{k}\right) \Delta v_{k}+\frac{\alpha g\left(\Delta u_{0}\right) \Delta v_{0}}{1-\alpha}, \\
& \Psi^{\prime}(u)(v)=\sum_{k=1}^{T} f\left(k, u_{k}\right) v_{k},
\end{aligned}
$$

for all $u, v \in S$. It is clear that

$$
\begin{aligned}
\sum_{k=0}^{T} g\left(\Delta u_{k}\right) \Delta v_{k} & =\sum_{k=0}^{T} g\left(\Delta u_{k}\right) v_{k+1}-\sum_{k=0}^{T} g\left(\Delta u_{k}\right) v_{k} \\
& =\sum_{k=1}^{T} g\left(\Delta u_{k-1}\right) v_{k}-\sum_{k=1}^{T} g\left(\Delta u_{k}\right) v_{k}-g\left(\Delta u_{0}\right) v_{0} \\
& =-\sum_{k=1}^{T} \nabla\left(g\left(\Delta u_{k}\right)\right) v_{k}-\alpha g\left(\Delta u_{0}\right) v_{1} \\
& =-\sum_{k=1}^{T} \nabla\left(g\left(\Delta u_{k}\right)\right) v_{k}-\frac{\alpha g\left(\Delta u_{0}\right) \Delta v_{0}}{1-\alpha},
\end{aligned}
$$

then,

$$
\left[\Phi^{\prime}(u)-\lambda \Psi^{\prime}(u)\right](v)=-\sum_{k=1}^{T}\left[\nabla\left(g\left(\Delta u_{k}\right)\right)+\lambda f\left(k, u_{k}\right)\right] v_{k}
$$

Consequently, the critical points of $I_{\lambda}$ in $S$ are exactly the solutions of the following boundary value problem:

$$
\left\{\begin{array}{l}
\nabla\left(g\left(\Delta u_{k}\right)\right)+\lambda f\left(k, u_{k}\right)=0, \quad k \in Z(1, T), \\
u_{0}=\alpha u_{1}, u_{T+1}=0
\end{array}\right.
$$

Remark 2. If $u \in S$ is a solution of problem (20) with $\left|\Delta u_{k}\right|$ $\leq \sqrt{3} / 2$ for $k \in Z(0, T)$, then, $u$ is a solution of problem (1).

\section{Main Results}

First, we consider the existence of nontrivial solutions for problem (1). Let

$$
B:=\limsup _{t \rightarrow 0^{+}} \frac{\sum_{k=1}^{T} F(k, t)}{t^{2}}
$$

We have the following:

Theorem 3. Assume that there exist two real sequences $\left\{u_{n}\right\}$ and $\left\{v_{n}\right\}$, with $v_{n}>0$ and $\lim _{n \longrightarrow+\infty} v_{n}=0$, such that 


$$
\begin{gathered}
\frac{u_{n}^{2}}{1+\sqrt{1-u_{n}^{2}}}+\frac{(1-\alpha) u_{n}^{2}}{1+\sqrt{1-(1-\alpha)^{2} u_{n}^{2}}}<\frac{v_{n}^{2}}{2 T}, \quad n \in Z(1), \\
\gamma:=\liminf _{n \rightarrow \infty} \frac{\sum_{k=1}^{T} \max _{|t| \leq v_{n}} F(k, t)-\sum_{k=1}^{T} F\left(k, u_{n}\right)}{v_{n}^{2} / 2 T-\left(u_{n}^{2} /\left(1+\sqrt{1-u_{n}^{2}}\right)\right)-\left(\left((1-\alpha) u_{n}^{2}\right) /\left(1+\sqrt{1-(1-\alpha)^{2} u_{n}^{2}}\right)\right)}<\frac{2 B}{2-\alpha} .
\end{gathered}
$$

Then, for each $\lambda \in((2-\alpha) / 2 B, 1 / \gamma)$, problem (1) admits a sequence of nontrivial solutions which converges to zero.

Remark 4. A sequence $\left\{u^{(n)}\right\}$ in $S$ is said to converge to zero if $\left\|u^{(n)}\right\| \longrightarrow 0$ as $n \longrightarrow \infty$.

Proof. Take $\Phi$ and $\Psi$ as defined by (14); we will prove Theorem 3 by using Lemma 1 . Since $\lim _{|s| \longrightarrow+\infty} G(s)=$ $\lim _{|s| \longrightarrow+\infty} s^{2}-(1 / 4)=+\infty$, it is easy to see that $\lim _{\|u\| \longrightarrow+\infty}$ $\Phi(u)=+\infty$, and $(\Lambda)$ is satisfied. Put

$$
r_{n}=\frac{v_{n}^{2}}{2 T}
$$

Since $\lim _{n \rightarrow \infty} v_{n}=0$, there is no harm in assuming that $v_{n} \leq \sqrt{T}$; then, $r_{n} \leq 1 / 2$. If $u \in S$ and

$$
\Phi(u)=\sum_{k=0}^{T} G\left(\Delta u_{k}\right)+\frac{\alpha G\left(\Delta u_{0}\right)}{1-\alpha}<r_{n}
$$

then, $G\left(\Delta u_{k}\right)<(1 / 2)$ for $k \in Z(0, T)$. Noting that $G(s) \geq 1 / 2$ for $|s| \geq \sqrt{3} / 2$, we see that $\left|\Delta u_{k}\right|<\sqrt{3} / 2$ for $k \in Z(0, T)$ and $\Phi(u)$ takes the form

$$
\Phi(u)=\sum_{k=0}^{T} \frac{\left(\Delta u_{k}\right)^{2}}{1+\sqrt{1-\left(\Delta u_{k}\right)^{2}}}+\frac{\alpha(1-\alpha) u_{1}^{2}}{1+\sqrt{1-(1-\alpha)^{2} u_{1}^{2}}}
$$

Therefore,

$$
\frac{1}{2} \sum_{k=1}^{T}\left(\Delta u_{k}\right)^{2} \leq \Phi(u)<r_{n}
$$

which implies that $\|u\|<\sqrt{2 r_{n}}$. By (11), we have

$$
\|u\|_{\infty}<\sqrt{2 T r_{n}}=v_{n} .
$$

According to the definition of $\mu$, we have

$$
\mu\left(r_{n}\right) \leq \inf _{u \in \Phi^{-1}\left(-\infty, r_{n}\right)} \frac{\sum_{k=1}^{T} \max _{|t| \leq v_{n}} F(k, t)-\sum_{k=1}^{T} F\left(k, u_{k}\right)}{\left(v_{n}^{2} / 2 T\right)-\Phi(u)} .
$$

For each $n \in Z(1)$, let $w_{n} \in S$ be defined by $\left(w_{n}\right)_{k}=u_{n}$ for every $k \in Z(1, T)$ and $\left(w_{n}\right)_{0}=\alpha\left(w_{n}\right)_{1},\left(w_{n}\right)_{T+1}=0$. Then,

$$
\Phi\left(w_{n}\right)=\frac{u_{n}^{2}}{1+\sqrt{1-u_{n}^{2}}}+\frac{(1-\alpha) u_{n}^{2}}{1+\sqrt{1-(1-\alpha)^{2} u_{n}^{2}}}<\frac{v_{n}^{2}}{2 T}=r_{n}
$$

by using (22). Thus,

$$
\mu\left(r_{n}\right) \leq \frac{\sum_{k=1}^{T} \max _{|t| \leq v_{n}} F(k, t)-\sum_{k=1}^{T} F\left(k,\left(w_{n}\right)_{k}\right)}{\left(v_{n}^{2} / 2 T\right)-\Phi\left(w_{n}\right)}=\frac{\sum_{k=1}^{T} \max _{|t| \leq v_{n}} F(k, t)-\sum_{k=1}^{T} F\left(k, u_{n}\right)}{\left(v_{n}^{2} / 2 T\right)-\left(u_{n}^{2} /\left(1+\sqrt{1-u_{n}^{2}}\right)\right)-\left(\left((1-\alpha) u_{n}^{2}\right) /\left(1+\sqrt{1-(1-\alpha)^{2} u_{n}^{2}}\right)\right)}
$$

Therefore, by (23), we know that $\delta \leq \liminf _{n \longrightarrow+\infty} \mu\left(r_{n}\right)$ $\leq \gamma<+\infty$.

To get our results, we need to show that conclusion $\left(a_{2}\right)$ of Lemma 1 holds. Therefore, we want to show that the global minimum $u \equiv 0$ of $\Phi$ is not a local minimum of $I_{\lambda}$. To prove this, we consider two cases: $B=+\infty$ and $B<+\infty$. In the case where $B=+\infty$, let $\left\{t_{n}\right\}$ be a sequence of positive numbers, with $t_{n} \in(0, \sqrt{3} / 2)$ and $\lim _{n \longrightarrow+\infty} t_{n}$ $=0$, such that 


$$
\sum_{k=1}^{T} F\left(k, t_{n}\right) \geq \frac{3 t_{n}^{2}}{\lambda}, \quad n \in Z(1) .
$$

Defining a sequence $\left\{\theta_{n}\right\}$ in $S$ by $\left(\theta_{n}\right)_{k}=t_{n}$ for $k \in Z$ $(1, T)$ and $\left(\theta_{n}\right)_{0}=\alpha\left(\theta_{n}\right)_{1},\left(\theta_{n}\right)_{T+1}=0$, we have

$$
\begin{aligned}
I_{\lambda}\left(\theta_{n}\right) & =\frac{t_{n}^{2}}{1+\sqrt{1-t_{n}^{2}}}+\frac{(1-\alpha) t_{n}^{2}}{1+\sqrt{1-(1-\alpha)^{2} t_{n}^{2}}}-\lambda \sum_{k=1}^{T} F\left(k, t_{n}\right) \\
& =-(1+\alpha) t_{n}^{2}<0 .
\end{aligned}
$$

In the case where $B<+\infty$, since $\lambda>(2-\alpha) / 2 B$, we can choose a small number $\varepsilon \in(0, \sqrt{3} / 2)$ such that

$$
\frac{1}{1+\sqrt{1-\varepsilon^{2}}}+\frac{(1-\alpha)}{1+\sqrt{1-(1-\alpha)^{2} \varepsilon^{2}}}<\lambda(B-\varepsilon) .
$$

By the definition of $B$, we can find a sequence of real numbers $\left\{\tau_{n}\right\}$ with $\tau_{n} \in(0, \varepsilon)$ such that $\lim _{n \longrightarrow+\infty} \tau_{n}=0$ and

$$
\sum_{k=1}^{T} F\left(k, \tau_{n}\right) \geq(B-\varepsilon) \tau_{n}^{2}
$$

Defining a sequence $\left\{\xi_{n}\right\}$ in $S$ by $\left(\xi_{n}\right)_{k}=\tau_{n}$ for $k \in Z$ $(1, T)$ and $\left(\xi_{n}\right)_{0}=\alpha\left(\xi_{n}\right)_{1},\left(\xi_{n}\right)_{T+1}=0$, we have

$$
\begin{aligned}
I_{\lambda}\left(\xi_{n}\right) & =\frac{\tau_{n}^{2}}{1+\sqrt{1-\tau_{n}^{2}}}+\frac{(1-\alpha) \tau_{n}^{2}}{1+\sqrt{1-(1-\alpha)^{2} \tau_{n}^{2}}}-\lambda \sum_{k=1}^{T} F\left(k, \tau_{n}\right) \\
& \leq\left(\frac{1}{1+\sqrt{1-\varepsilon^{2}}}+\frac{(1-\alpha)}{1+\sqrt{1-(1-\alpha)^{2} \varepsilon^{2}}}-\lambda(B-\varepsilon)\right) \tau_{n}^{2} \\
& <0 .
\end{aligned}
$$

Noticing that $I_{\lambda}(0)=0$, we see that $u \equiv 0$ is not a local minimum of $I_{\lambda}$ by combining the above two cases. Therefore, by Lemma 1 and Remark 2, we know the conclusion of Theorem 3 holds.

Now, let

$$
B_{*}=\liminf _{t \longrightarrow 0^{+}} \frac{\sum_{k=1}^{T} \max _{|s| \leq t} F(k, s)}{t^{2}} .
$$

Then, there exists a sequence $\left\{v_{n}\right\}$ of positive numbers with $\lim _{n \longrightarrow+\infty} v_{n}=0$ such that

$$
\lim _{n \longrightarrow \infty} \frac{\sum_{k=1}^{T} \max _{|t| \leq v_{n}} F(k, t)}{v_{n}^{2}}=B_{*} .
$$

Taking $u_{n}=0$ for all $n \in Z(1)$, by Theorem 3 , we get the following corollary.

Corollary 5. If

$$
T B_{*}<\frac{B}{2-\alpha}
$$

Then, for each $\lambda \in\left((2-\alpha) / 2 B, 1 / 2 T B_{*}\right)$, problem (1) admits a sequence of nontrivial solutions which converges to zero.

To obtain the positive solutions of problem (20), we need the following strong maximum principle.

Theorem 6. Assume $u \in S$ such that either

$$
u_{k}>0 \operatorname{or} \nabla\left(g\left(\Delta u_{k}\right)\right) \leq 0
$$

for all $k \in Z(1, T)$. Then, either $u_{k}>0$ for all $k \in Z(1, T)$ or $u \equiv 0$.

Proof. There exists $\tau \in Z(1, T)$ such that

$$
u_{\tau}=\min \left\{u_{k}: k \in N(1, T)\right\}
$$

If $u_{\tau}>0$, then $u_{k}>0$ for all $k \in Z(1, T)$ and the proof is complete.

If $u_{\tau} \leq 0$, then $u_{\tau}=\min \left\{u_{k}: k \in N(0, T+1)\right\}$. Because $\Delta u_{\tau-1}=u_{\tau}-u_{\tau-1} \leq 0$ and $\Delta u_{\tau}=u_{\tau+1}-u_{\tau} \geq 0, \quad g(s)$ is increasing in $s$, and $g(0)=0$, we have

$$
g\left(\Delta u_{\tau}\right) \geq 0 \geq g\left(\Delta u_{\tau-1}\right)
$$

On the other hand, let $k=\tau$; (40) implies

$$
g\left(\Delta u_{\tau}\right) \leq g\left(\Delta u_{\tau-1}\right)
$$

By combining (42) with (43), we get $g\left(\Delta u_{\tau}\right)=0=g(\Delta$ $\left.u_{\tau-1}\right)$. That is $u_{\tau+1}=u_{\tau-1}=u_{\tau}$. If $\tau+1=T+1$, we have $u_{\tau}$ $=0$. Otherwise, $\tau+1 \in N(1, T)$. Replacing $\tau$ by $\tau+1$, we get $u_{\tau+2}=u_{\tau+1}$. Continuing this process $T+1-\tau$ times, we have $u_{\tau+j}=0$ for $j \in Z(0, T+1-\tau)$. Similarly, we have $u_{j}=$ 0 for $j \in Z(0, \tau)$. Thus, $u \equiv 0$ and the proof is complete.

Now, we are ready to establish the existence of positive solutions for problem (1); we have

Corollary 7. If $f(k, 0) \geq 0$ for all $k \in Z(1, T)$,

$$
A_{*}:=\liminf _{t \longrightarrow 0^{+}} \frac{2 T \sum_{k=1}^{T} \max _{0 \leq s \leq t} \int_{0}^{s} f(k, x) d x}{t^{2}}<\frac{2 B}{2-\alpha} .
$$

Then, for each $\lambda \in\left((2-\alpha) / 2 B, 1 / A_{*}\right)$, problem (1) admits a sequence of positive solutions which converges to zero. 
Proof. Put

$$
f^{*}(k, x)= \begin{cases}f(k, x), & \text { if } x>0, \\ f(k, 0), & \text { if } x \leq 0 .\end{cases}
$$

Noticing that $f(k, 0) \geq 0$, we see that

$$
\max _{0 \leq|s| \leq t} \int_{0}^{s} f^{*}(k, x) d x=\max _{0 \leq s \leq t} \int_{0}^{s} f(k, x) d x,
$$

for all $t \geq 0$. By Corollary 5, we know that problem (1) with $f$ replaced by $f^{*}$ admits a sequence of nontrivial solutions which converges to zero for each $\lambda \in\left((2-\alpha) / 2 B, 1 / A_{*}\right)$. And by Theorem 6, we know that all these solutions are positive.

\section{An Example}

In this section, we give an example to illustrate our main results.

Example 8. Consider the boundary value problem (1) with

$f(k, x)=f(x)=\left\{\begin{array}{l}x(2+2 \varepsilon+2 \sin (\varepsilon \ln |x|)+\varepsilon \cos (\varepsilon \ln |x|)), \quad x \neq 0, \\ 0, \quad x=0,\end{array}\right.$

for $k \in Z(1, T)$. Then,

$F(k, x)=F(x)=\int_{0}^{x} f(s) d s=x^{2}(1+\varepsilon+\sin (\varepsilon \ln x)), \quad$ for $x>0$.

Since $f(x) \geq 0$ for $x \geq 0$, we see that $F(x)$ is increasing in $x \in[0,+\infty)$. Thus,

$$
\begin{aligned}
A_{*} & :=\liminf _{t \longrightarrow 0^{+}} \frac{2 T \sum_{k=1}^{T} \max _{0 \leq s \leq t} \int_{0}^{s} f(k, x) d x}{t^{2}} \\
& =\liminf _{t \longrightarrow 0^{+}} 2 T^{2}(1+\varepsilon+\sin (\varepsilon \ln t))=2 T^{2} \varepsilon, \\
B & :=\limsup _{t \longrightarrow 0^{+}} \frac{\sum_{k=1}^{T} F(k, t)}{t^{2}} \\
& =\limsup _{t \longrightarrow 0^{+}} T(1+\varepsilon+\sin (\varepsilon \ln t)) \\
& =T(2+\varepsilon) .
\end{aligned}
$$

Let $\varepsilon \in(0,2 /((2 T-\alpha T-1)))$; then (44) holds. By Corollary 7 , for each $\lambda \in\left((2-\alpha) /(2 T(2+\varepsilon)), 1 / 2 T^{2} \varepsilon\right)$, problem (1) admits a sequence of positive solutions which converges to zero.

\section{Conclusions}

In this paper, we consider a discrete boundary value problem involving the singular $\phi$-Laplacian. The problem contains the Dirichlet problem (when $\alpha=0$ ) and Robin problem (when $\alpha=1$ ) as special cases. Since the domain of the singular operator $\phi$ is $(-1,1)$, we can not apply the critical point theory to this problem directly. Therefore, we extend the domain of the singular operator to the whole real numbers and consider an auxiliary problem associated with the original one. The conditions for the multiplicity of positive solutions of the discrete boundary problem are found, and an illustrative example is given. The method in this paper provides a new way to discuss the boundary value problems containing a singular Laplacian.

\section{Data Availability}

No data were used to support the study.

\section{Conflicts of Interest}

The author declares that he has no conflicts of interest.

\section{Acknowledgments}

This work is supported by the National Natural Science Foundation of China (Grant No. 11571084).

\section{References}

[1] T. Chen, R. Ma, and Y. Liang, "Multiple positive solutions of second-order nonlinear difference equations with discrete singular $\varphi$-Laplacian," Journal of Difference Equations and Applications, vol. 25, no. 1, pp. 38-55, 2019.

[2] C. Bereanu and J. Mawhin, "Boundary value problems for second-order nonlinear difference equations with discrete $\varphi$ Laplacian and singular $\varphi$," Journal of Difference Equations and Applications, vol. 14, no. 10-11, pp. 1099-1118, 2008.

[3] A. E. Treibergs, "Entire spacelike hypersurfaces of constant mean curvature in Minkowski space," Inventiones Mathematicae, vol. 66, no. 1, pp. 39-56, 1982.

[4] C. Bereanu, P. Jebelean, and P. Torres, "Positive radial solutions for Dirichlet problems with mean curvature operators in Minkowski space," Journal of Functional Analysis, vol. 264, no. 1, pp. 270-287, 2013

[5] S. Boulaaras, Y. Bouizem, and R. Guefaifia, "Further results of existence of positive solutions of elliptic Kirchhoff equation with general nonlinearity of source terms," Mathematical Methods in the Applied Sciences, vol. 43, no. 15, pp. 91959205, 2020.

[6] M. Haiour, S. Boulaaras, R. Guefaifia, and F. Kamache, "Existence result for a new class of Kirchhoff elliptic system with variable parameters," Miskolc Mathematical Notes, vol. 21, no. 2, pp. 887-896, 2020.

[7] S. Boulaaras, R. Guefaifia, B. Cherif et al., "Existence result for a Kirchhoff elliptic system involving p-Laplacian operator with variable parameters and additive right hand side via sub and super solution methods," AIMS Mathematics, vol. 6, no. 3, pp. 2315-2329, 2021.

[8] M. Haiour, S. Boulaaras, Y. Bouizem, and R. Guefaifia, "Existence result for a Kirchhoff elliptic system with variable parameters and additive right-hand side via sub- and supersolution method," Boundary Value Problems, vol. 2020, no. 1, 2020 . 
[9] S. Boulaaras, "Existence of positive solutions for a new class of Kirchhoff parabolic systems," Rocky Mountain Journal of Mathematics, vol. 50, no. 2, pp. 445-454, 2020.

[10] R. P. Agarwal, D. O’Regan, and J. Y. P. Wong, Positive Solutions of Differential, Difference and Integral Equations, Kluwer Academic Publishers, Dordrecht, 1999.

[11] J. Henderson and H. B. Thompson, "Existence of multiple solutions for second-order discrete boundary value problems," Computers \& Mathematics with Applications, vol. 43, no. 1011, pp. 1239-1248, 2002.

[12] Z. M. Guo and J. S. Yu, "Existence of periodic and subharmonic solutions for second-order superlinear difference equations," Science in China. Series A. Mathematics, vol. 46, no. 4, pp. 506-515, 2003.

[13] J. Mawhin, "Periodic solutions of second order nonlinear difference systems with $\phi$-Laplacian: a variational approach," Nonlinear Analysis, vol. 75, no. 12, pp. 4672-4687, 2012.

[14] H. Shi, "Periodic and subharmonic solutions for second-order nonlinear difference equations," Journal of Applied Mathematics and Computing, vol. 48, no. 1-2, pp. 157-171, 2015.

[15] Z. Zhou, J. S. Yu, and Y. M. Chen, "Homoclinic solutions in periodic difference equations with saturable nonlinearity," Science China Mathematics, vol. 54, no. 1, pp. 83-93, 2011.

[16] Z. Zhou and J. S. Yu, "Homoclinic solutions in periodic nonlinear difference equations with superlinear nonlinearity," Acta Mathematica Sinica-English Series, vol. 29, no. 9, pp. 1809-1822, 2013.

[17] Z. Zhou and D. F. Ma, "Multiplicity results of breathers for the discrete nonlinear Schrödinger equations with unbounded potentials," Science China Mathematics, vol. 58, no. 4, pp. 781-790, 2015.

[18] G. H. Lin and Z. Zhou, "Homoclinic solutions of discrete $\$ \backslash$ phi\$-Laplacian equations with mixed nonlinearities," Communications on Pure \& Applied Analysis, vol. 17, no. 5, pp. 1723-1747, 2018.

[19] G. H. Lin, Z. Zhou, and J. S. Yu, "Ground state solutions of discrete asymptotically linear Schrödinger equations with bounded and non-periodic potentials," Journal of Dynamics and Differential Equations, vol. 32, no. 2, pp. 527-555, 2020.

[20] X. H. Tang, "Non-Nehari manifold method for periodic discrete superlinear Schrödinger equation," Acta Mathematica Sinica-English Series, vol. 32, no. 4, pp. 463-473, 2016.

[21] Q. Q. Zhang, "Homoclinic orbits for a class of discrete periodic Hamiltonian systems," Proceedings of the American Mathematical Society, vol. 143, no. 7, pp. 3155-3163, 2015.

[22] Q. Q. Zhang, "Homoclinic orbits for discrete Hamiltonian systems with local super-quadratic conditions," Communications on Pure \& Applied Analysis, vol. 18, no. 1, pp. 425-434, 2019.

[23] J. H. Kuang and Z. M. Guo, "Heteroclinic solutions for a class of p-Laplacian difference equations with a parameter," Applied Mathematics Letters, vol. 100, p. 106034, 2020.

[24] Z. Zhou and M. T. Su, "Boundary value problems for 2 n-order $\phi c$-Laplacian difference equations containing both advance and retardation," Applied Mathematics Letters, vol. 41, no. 1, pp. 7-11, 2015.

[25] G. Bonanno and P. Candito, "Infinitely many solutions for a class of discrete non-linear boundary value problems," Applicable Analysis, vol. 88, no. 4, pp. 605-616, 2009.

[26] G. Bonanno, P. Jebelean, and C. Serban, "Superlinear discrete problems," Applied Mathematics Letters, vol. 52, no. 1, pp. 162-168, 2016.
[27] G. D'Aguì, J. Mawhin, and A. Sciammetta, "Positive solutions for a discrete two point nonlinear boundary value problem with $p$-Laplacian," Journal of Mathematical Analysis and Applications, vol. 447, no. 1, pp. 383-397, 2017.

[28] Z. Zhou and J. X. Ling, "Infinitely many positive solutions for a discrete two point nonlinear boundary value problem with $\phi c$ Laplacian," Applied Mathematics Letters, vol. 91, no. 1, pp. 2834, 2019.

[29] J. X. Ling and Z. Zhou, "Positive solutions of the discrete Dirichlet problem involving the mean curvature operator," Open Mathematics, vol. 17, no. 1, pp. 1055-1064, 2019.

[30] J. X. Ling and Z. Zhou, "Positive solutions of the discrete Robin problem with \$\phi\$-Laplacian," Discrete and Continuous Dynamical Systems. Series S, vol. 14, no. 9, pp. 3183-3196, 2021.

[31] B. Ricceri, "A general variational principle and some of its applications," Journal of Computational and Applied Mathematics, vol. 113, no. 1-2, pp. 401-410, 2000.

[32] R. P. Agarwal, Difference Equations and Inequalities: Theory, Methods, and Applications, Marcel Dekker Inc., New York, NY, USA, 2000.

[33] W. G. Kelly and A. C. Peterson, Difference Equations: An Introduction with Applications, Academic Press, San Diego, CA, USA, 1991. 